\title{
Anthropometric and Metabolic Characteristics of Overweight and Normal Weight Preschoolers: a Cross-Sectional Study
}

\author{
Kathleen E Bethin ${ }^{1 *}$, Lisa Rafalson', Paul Creighton ${ }^{2}$, Husam Ghanim³ ${ }^{3}$ Paresh \\ Dandona $^{3}$, Amy Stone Nagai ${ }^{2}$ and Teresa Quattrin ${ }^{1}$ \\ ${ }^{1}$ Pediatrics, Division of Endocrinology/Diabetes, University at Buffalo School of Medicine \& Biomedical Sciences, \\ Women and Children's Hospital of Buffalo, New York, USA \\ ${ }^{2}$ Pediatric \& Community Dentistry, University at Buffalo School of Dental Medicine, Women and Children's Hospital \\ of Buffalo, New York, USA \\ ${ }^{3}$ Division of Endocrinology, Diabetes and Metabolism, University at Buffalo School of Medicine \& Biomedical \\ Sciences, New York, USA
}

Received: January 01, 2015; Accepted: February 21, 2015; Published: March 16, 2015

*Corresponding author: Kathleen E. Bethin, University at Buffalo School of Medicine \& Biomedical Sciences, Women and Children's Hospital of Buffalo, 219 Bryant St., Buffalo, NY, USA 14222,Tel: 17168787588; Fax: 17168883827; E-mail: kbethin@upa.chob.edu

\begin{abstract}
Objective: The aims of this study were to determine metabolic characteristics of normal weight and overweight 2-5 year old children and to collect data pertinent to energy intake.

Design and methods: Study participants (2-5years) $(n=103)$ were recruited when they arrived for full mouth rehabilitation under general anesthesia. Height and weight were measured. After induction of anesthesia, waist circumference was measured and fasting bloods drawn (lipids, glucose, insulin and CRP). BMI, WCHtR and HOMAIR were calculated. Metabolic and anthropometric characteristics were compared between normal and overweight children. Statistical comparisons were performed using two-sided t-tests or chi-square test. Block food frequency questionnaires were completed by the caretakers.
\end{abstract}

Results: $34.4 \%(n=33)$ of participants were overweight. WCHtR was 0.05 units higher and both LDL and total cholesterol were high in the overweight group. WCHtR positively correlated with BMI z-score, WC, HOMA-IR, insulin, glucose, and CRP $(r=0.75,0.67,0.31,0.29,0.25$, $0.32,0.33$, respectively) and negatively correlated with HDL ( $\mathrm{r}=-0.30)$.

Conclusions: Among 2-5 year old with poor dental health, the prevalence of BMI $\geq 85^{\text {th }}$ percentile is very high and abnormalities in cardiovascular risk factors are already present. To our knowledge, this is the first study demonstrating an association between waist circumference to height ratio and CRP and insulin resistance in children less than 5 years. Overweight preschoolers should be screened for associated cardiovascular markers such as abnormal lipid profile.

Keywords: Lipid panel; Preschoolers; Pediatrics; Body Mass Index; Obesity

\author{
Abbreviations \\ BMI: Body Mass Index; CRP: C -reactive protein; dmft: \\ decayed, missing (due to caries) and filled teeth; ELISA: Enzyme-
}

Linked Immunosorbent Assay; HDL-C: High-Density Lipoprotein Cholesterol; HOMA-IR: Homeostatic Model Assessment- Insulin Resistance; LDL-C: Low-Density Lipoprotein Cholesterol; SD: Standard Deviation; WCHtR: Waist Circumference to Height Ratio

\section{Introduction}

For the past 30 years, the prevalence of obesity in the United States has been increasing [1]. Data from NHANES 2007-08 demonstrate that among children aged 2-19 years the prevalence of overweight or obesity is $34.7 \%$ and in $2-5$ year olds is $21.2 \%[2,3]$. The increase in obesity observed in children is likely a contributing factor to the rising incidence of type 2 diabetes mellitus and related co-morbidities in youth $[3,4]$. It is not known how early in life obesity may lead to increased risk for what are traditionally considered "adult" co-morbidities such as hypertension, cardiovascular disease, and type 2 diabetes mellitus. Obesity among American Indian children and adolescents has been associated with increased mortality in adulthood [5]. Despite this alarmingly high prevalence of obesity in children, very little is known about effects of obesity on the metabolic parameters of young children. A number of studies have looked at parameters related to the metabolic syndrome in children, but have all been done in children older than the preschool age. In one study of 370 youth aged 6-14 years presenting to a lipid clinic in Rome, Italy, BMI, mid-upper arm circumference, hip circumference and waist circumference were all significantly higher in the hypertensive group (19.5\% of the attendees) [6]. In adults, multiple large studies have found a direct relationship of high LDL-cholesterol or total cholesterol and both new onset coronary heart disease and recurrent disease $[7,8]$. Elevated LDL-cholesterol in children has been demonstrated to correlate with atherosclerotic disease in children and young adults who died in accidents [9]. Guidelines for normal lipids in children 2-19 years of age have been published by the National 
Cholesterol Education Program Expert Panel, yet they do not take into account the normal age-dependent differences that occur during growth and development [10-12]. In addition, the data driving these recommendations do not include LDL-cholesterol or HDL-cholesterol for children under 5 years of age [13]. This is due in part to the fact that young children rarely need routine blood work and parents of preschoolers are unwilling to submit their children to venipuncture for a research study. Given these limitations, we chose a convenient sample of preschool children for this study who required intravenous access and general anesthesia for full mouth rehabilitation. The primary aim of our study was to collect data in this selected sample to relate anthropometric parameters to cardiovascular risk markers, specifically lipid panels and insulin resistance (measured by HOMA-IR). Since energy intake and macronutrients can affect lipid profiles and insulin levels [14], our secondary aim was to collect data pertinent to energy and macronutrient intake and examine a possible association between energy/ macronutrient intake and lipid profile and HOMA-IR.

\section{Methods}

\section{Study plan and members}

Participants: Potential study participants were identified in a sequential manner over a six month period from June December, 2009 by the Pediatric Dentist (PC) when they arrived at Women and Children's Hospital of Buffalo for full mouth rehabilitation under general anesthesia for varying degrees of dental decay. We included: 2-5 year old females and males, who had an English-speaking parent/guardian. We excluded children with Body Mass Index (BMI) $<5^{\text {th }}$ percentile as under nutrition may indicate an underlying chronic disorder. The study was approved by the Children and Youth Institutional Review Board. Only three out of 108 potential participants refused to participate and two could not participate due to parental language barrier.

Patients presented in early morning having fasted for 8-12 hours and underwent a physical exam performed by a nurse practitioner. Height was measured to the nearest $0.5 \mathrm{~cm}$ using a dedicated stadiometer (calibrated monthly) and weight was measured with child in a hospital gown to the nearest $0.5 \mathrm{~kg}$ using a Tanita BWB-800 scale calibrated with a 25 pound weight by one of the investigators (KB) or a trained research assistant in the operating room. BMI was calculated as weight $(\mathrm{kg}) / \mathrm{height}\left(\mathrm{m}^{2}\right)$. After induction with nitrous oxide, a blood sample was drawn and supine waist circumference was obtained to the nearest $0.1 \mathrm{~cm}$ by flexible tape at the level of the umbilicus. Waist circumference $(\mathrm{cm})$ to height $(\mathrm{cm})$ ratio $(\mathrm{WCHtR})$ was calculated. Blood was spun within $30^{\prime}-60^{\prime}$ and frozen at $-70^{\circ} \mathrm{C}$ until assayed. Nutritional data were collected using the Block Kids questionnaire (ages 2-7 years), filled out by the accompanying parent /guardian while the child was in the operating room [15-17].

\section{Materials and procedures}

Laboratory analysis: Lipid profile was measured by Kaleida Health Laboratory and CRP, insulin and blood glucose were assayed by the Diabetes/Endocrinology Research
Laboratory at the University at Buffalo. Glucose was measured via glucoseoxidase-02 electrode and insulin and CRP were measured by ELISA (Diagnostic Systems Laboratories Inc., Webster, TX). Total cholesterol was determined enzymatically with the Beckman Coulter reagent. HDL-C was determined using a Beckman Coulter reagent via a homogenous (direct) polyanion method using detergent and cholesterol esterase/oxidase. Triglyceride levels were determined enzymatically using glycerol phosphate oxidase without correction for free glycerol. LDL-C was calculated using the Friedewald equation. HOMA-IR was calculated; [glucose (mg/dl) x insulin $(\mu \mathrm{U} / \mathrm{ml})] / 405$ [18].

Data analysis: Statistical analyses were performed on 96 subjects. Normal weight was defined as BMI $5^{\text {th }}$ to $<85^{\text {th }}$ percentile and overweight or obese as $\mathrm{BMI} \geq 85^{\text {th }}$ percentile for age and gender. Data were expressed as mean \pm SD or percentage. Statistical comparisons were performed using t-tests or chisquare test as applicable. All tests were two-sided and a p-value less than 0.05 were considered statistically significant. The decayed missing (due to caries) and filled teeth (dmft) score was used. A similar scoring system (DMFT) is used to assess permanent teeth [19], but the dmft score is used to assess caries in the primary teeth of young children [20-25]. Patient charts were reviewed by A. Nagai and dmft score was calculated from the dental exam done at the time of dental surgery by a single pediatric dentist (P. Creighton). A score of 0 indicates that none of the teeth have damage while a maximum score of 20 indicates that $100 \%$ of teeth are affected.

\section{Results}

Table 1 illustrates the demographic and anthropometric characteristics of the participating children as a whole and divided based on a BMI above and below the $85^{\text {th }}$ percentile. In this population with poor dental health $34.4 \%$ of study participants were overweight or obese. As expected mean waist circumference and WCHtR were significantly higher in the overweight or obese versus normal weight youth. While we did not collect specific data pertinent to socioeconomic status, 75\% were covered by Medicaid, managed Medicaid and Fidelis (New York State-sponsored health insurance) and only 25\% had commercial insurance. There was no difference in the prevalence of overweight or obesity between children with commercial versus state-sponsored coverage (Table 1). The dmft score did not differ between children with normal weight or overweight (Table 1) and did not show a correlation with BMI z-score, anthropometric or laboratory measurements. However, the $\mathrm{dmft}$ score was very high in this population with mean 10.0 , median 10.0 and range $3-16$.

Table 2 illustrates the results of the metabolic work-up. Total and LDL cholesterol were higher in the overweight or obese group ( $p=0.04$ ). Total cholesterol was above $170 \mathrm{mg} / \mathrm{dL}$ in $19 \%$ of the normal weight and $30 \%$ of the overweight or obese children. Similarly, the LDL-cholesterol was above $110 \mathrm{mg} / \mathrm{dL}$ in $14 \%$ of the normal weight and $36 \%$ of the overweight or obese children. CRP did not differ between groups and ranged from 0.023 to 6.0 $\mathrm{mg} / \mathrm{L}$. While there was no significant difference in CRP, insulin, 
Table 1: Patient Demographic and Anthropometric Characteristics.

\begin{tabular}{|c|c|c|c|c|}
\hline Characteristic & All & $\begin{array}{l}\text { BMI } \\
5^{\text {th }}-<85^{\text {th }} \text { percentile }\end{array}$ & $\begin{array}{l}\text { BMI } \geq 85^{\text {th }} \\
\text { Percentile }\end{array}$ & p-value \\
\hline n & 96 & 63 & 33 & \\
\hline Age (years) & $4.1 \pm 1.1$ & $4.1 \pm 1.1$ & $4.1 \pm 1.1$ & 0.9 \\
\hline $\mathrm{M} / \mathrm{F}(\mathrm{n})$ & $49 / 47$ & $35 / 28$ & $14 / 19$ & \\
\hline Medicaid, \% & 75 & 71.4 & 75.8 & 0.3 \\
\hline Race/Ethnicity, n & & & & 0.2 \\
\hline Non-Hispanic White & 46 & 27 & 19 & \\
\hline African American & 31 & 24 & 7 & \\
\hline Hispanic & 6 & 4 & 2 & \\
\hline Other & 7 & 3 & 4 & \\
\hline Height $(\mathrm{cm})$ & $103.4 \pm 8.9$ & $102.6 \pm 8.2$ & $105.0 \pm 10$ & 0.2 \\
\hline Weight (kg) & $18.2 \pm 4.2$ & $16.5 \pm 2.5$ & $21.4 \pm 4.9$ & $<0.001$ \\
\hline BMI $\left(\mathrm{kg} / \mathrm{m}^{2}\right)$ & $16.91 \pm 2.5$ & $15.63 \pm 0.87$ & $19.34 \pm 2.84$ & $<0.001$ \\
\hline BMI z-score & $0.66 \pm 1.2$ & $-0.036 \pm 0.68$ & $2.00 \pm 0.90$ & $<0.001$ \\
\hline WCHtR & $0.48 \pm 0.050$ & $0.46 \pm 0.030$ & $0.51 \pm 0.060$ & $<0.001$ \\
\hline Waist circumference (cm) & $49.2 \pm 5.6$ & $46.6 \pm 2.6$ & $54.1 \pm 6.5$ & $<0.001$ \\
\hline dmft score & $10.0 \pm 2.7$ & $10.3 \pm 2.8$ & $9.06 \pm 2.7$ & 0.3 \\
\hline
\end{tabular}

Data are presented as mean $\pm \mathrm{SD}$, number $(\mathrm{n})$ or $\%$ of total. Two-sided t-test or chi-square test was performed as appropriate. $P$-value $<0.05$ was considered significant and represented in bold. BMI = Body Mass Index, WCHtR = Waist Circumference to Height Ratio

Table 2: Metabolic Profile.

\begin{tabular}{|c|c|c|c|c|}
\hline Parameter & All & $\begin{array}{l}\text { BMI } \\
5^{\text {th }}<<5^{\text {th }} \text { percentile }\end{array}$ & $\begin{array}{l}\text { BMI } \geq 85^{\text {th }} \\
\text { Percentile }\end{array}$ & $p$-value \\
\hline $\mathrm{n}$ & 96 & 63 & 33 & \\
\hline $\begin{array}{l}\text { CRP mg/L } \\
\text { CRP level>1.0 mg/L (\%) }\end{array}$ & $\begin{array}{l}0.73 \pm 1.2 \\
18.8\end{array}$ & $\begin{array}{l}0.71 \pm 1.2 \\
19.0\end{array}$ & $\begin{array}{l}0.75 \pm 1.2 \\
18.2\end{array}$ & $\begin{array}{l}0.9 \\
0.9\end{array}$ \\
\hline Blood glucose mg/dl & $86.7 \pm 12$ & $85.8 \pm 11$ & $88.3 \pm 13$ & 0.3 \\
\hline Blood glucose $\geq 100$ mg/dl (\%) & 10 & 10 & 12 & 0.7 \\
\hline Insulin $\mathrm{uU} / \mathrm{ml}$ & $2.0 \pm 1.5$ & $1.9 \pm 1.3$ & $2.1 \pm 1.8$ & 0.4 \\
\hline HOMA-IR & $0.43 \pm 0.35$ & $0.41 \pm 0.30$ & $0.48 \pm 0.43$ & 0.4 \\
\hline LDL-C mg/dl & $97.4 \pm 26$ & $93.6 \pm 19$ & $104.8 \pm 34$ & 0.04 \\
\hline Total cholesterol mg/dl & $150 \pm 29$ & $146 \pm 22$ & $159 \pm 37$ & 0.04 \\
\hline HDL-C mg/dl & $39.7 \pm 10$ & $39.9 \pm 11$ & $39.3 \pm 9.1$ & 0.8 \\
\hline Triglycerides mg/dl & $66 \pm 43$ & $63 \pm 36$ & $72 \pm 53$ & 0.3 \\
\hline
\end{tabular}

Data are presented as mean $\pm \mathrm{SD}$ or $\%$ of total. Two-sided t-test or chi-square test was performed as appropriate. $P$-value $<0.05$ was considered significant and represented in bold. $\mathrm{CRP}=\mathrm{C}-$ reactive protein, HOMA-IR $=$ Homeostasis Model of Assessment- Insulin Resistance, LDL-C $=$ LDLCholesterol, HDL-C = HDL-Cholesterol

glucose or HOMA-IR between groups, a positive correlation was observed between these parameters and WCHtR (Table 3). HDL level negatively correlated with WCHtR. Moreover a strong association was observed between BMI z-score and both waist circumference and the WCHtR (Table 3). We observed that WCHtR demonstrated a stronger correlation with a number of metabolic characteristics compared to the associations between these metabolic variables and the BMI z-score.

There was no difference in daily energy intake between the normal weight and overweight or obese children, $1591 \pm 692$ and $1469 \pm 561$ kilocalories, respectively. Overall, $51 \%$ of the total sample consumed more than 1400 kilocalories per day, 22\% consumed 1200-1400 kilocalories and only $27 \%$ reported eating less than 1200 kilocalories. Total daily kilocalories, daily intake of cholesterol, total carbohydrate, fructose, dairy and $100 \%$ juice consumed were positively associated with dmft score $(\mathrm{r}=0.22$, $0.25,0.28,0.24,0.25$ and 0.25 , respectively, all $p$-values $<0.050$ ).

\section{Discussion}

Data pertinent to the cardiovascular profile of youth with obesity are limited. Our study results show that 2-5 year old children with a $\mathrm{BMI} \geq 85^{\text {th }}$ percentile already have higher total 
Table 3: Pearson Correlations between BMI z-score, or Waist Circumference (WC) or WC to Height Ratio (WCHtR) and Metabolic Profile.

\begin{tabular}{|l|l|l|l|l|l|l|l|l|l|l|}
\hline & WC & WCHtR & dmft & HOMA-IR & Insulin & Glucose & CRP & TG & HDL \\
\hline $\begin{array}{l}\text { BMI } \\
\text { z-Score }\end{array}$ & $0.76^{* *}$ & $0.75^{* *}$ & 0.030 & $0.26^{*}$ & $0.24^{*}$ & 0.19 & $0.25^{*}$ & $0.22^{*}$ & -0.090 \\
\hline WC & & $0.67^{* *}$ & 0.030 & $0.42^{* *}$ & $0.41^{* *}$ & 0.14 & $0.30^{* *}$ & $0.35^{* *}$ & -0.11 \\
\hline WCHtR & & & -0.070 & $0.31^{* *}$ & $0.29^{* *}$ & $0.25^{*}$ & $0.32^{* *}$ & $0.33^{* *}$ & $-0.30^{* *}$ \\
\hline dmft & & & & 0.020 & 0.030 & 0.030 & 0.15 & -0.16 & -0.10 \\
\hline
\end{tabular}

${ }^{*} p<0.05$

${ }^{* *} p<0.005$

$\mathrm{BMI}=$ Body Mass Index, $\mathrm{WC}=$ Waist Circumference, $\mathrm{WCH} t \mathrm{R}=$ Waist Circumference to Height Ratio, dmft = decayed missing and filled primary teeth score

and LDL-cholesterol levels compared to children with normal weight. The mean total cholesterol and LDL-cholesterol in both groups of children was in the "acceptable range" for children [26]. However, the recommendations for "acceptable" lipid levels in children and adolescents are largely based on data from school-aged children and adolescents [10,12,13,27]. Therefore lower normative data may actually apply to younger youth. In addition to increased total and LDL-cholesterol with obesity, these young children demonstrated increased WCHtR, a surrogate marker of increased visceral fat, which has been shown to be associated with increased risk of cardiovascular disease in adults $[28,29]$. Among children aged 5 years and older in two separate studies from Italy and Bogalusa, LA observed a number of cardiovascular risk factors including higher blood pressure, triglycerides, LDL-cholesterol and fasting blood glucose in children with higher WCHtR [30,31]. In a study of 6-14 year old children from 14 towns in Calabria, Italy, the authors found a correlation between waist circumference to height ratio and triglyceride to HDL ratio [32]. In our population, the overweight or obese children are already demonstrating a WCHtR that is 0.05 units higher $(p<0.001)$ than the normal weight children with a positive correlation with CRP levels and HOMA-IR and a negative correlation with cardiovascular protective HDL. Skinner, et al. [33] demonstrated that in 3-5 year olds in the 1999-2006 NHANES dataset that hazard ratio for an elevated CRP was greatly increased in the very obese (BMI $\geq 99^{\text {th }}$ percentile) but did not see an increased hazard ratio in the simply obese children (BMI $\geq 95^{\text {th }}$ percentile) [33]. Mean \pm SD CRP levels in our patients was $0.73 \pm 1.2 \mathrm{mg} / \mathrm{L}$ compared to a mean of $0.89 \mathrm{mg} / \mathrm{L}$ in $1-17$ year olds from the 1999-2006 NHANES dataset [33]. In a smaller NHANES dataset, the CRP at the $50^{\text {th }}$ percentile for the 3-9 year age group was $0.3 \mathrm{mg} / \mathrm{L}$ [34]. Nevertheless, comparisons with NHANES data are made difficult by the fact that the age range of our population was younger. Our data suggest that in a population of 2-5 year olds with poor dental health increased waist circumference is already correlating with an unfavorable metabolic profile. Further, these data suggest that high WCHtR even at a very young age may already increase risk for insulin resistance and future cardiovascular disease. Further evidence of a link between high BMI and markers of cardiovascular risk are seen in data from older children that demonstrate that even a small reduction in BMI SDS of 0.5 units reduces waist circumference, insulin resistance, total and LDL-cholesterol [35].
Our data show that in this population of 2-5 year old children with poor dental health the prevalence of BMI $\geq 85^{\text {th }}$ percentile was $34.4 \%$, comparable or higher than national data [2]. Due to the fact that these children were fasting 8-12 hours, the percentage of overweight may have been underestimated due to decreased food and liquid intake. Using national data, socioeconomic status (SES) has been shown to be inversely related to childhood and adolescent obesity [36,37]. While we did not obtain data relative to SES, $75 \%$ of these children were covered by Medicaid or Managed Medicaid. One could therefore speculate that these children may have lower SES to explain the high prevalence of overweight in the sample studied.

A limitation of our study work is that participants were part of a selected population of children with poor dental health. Factors known to be associated with increased risk of dental caries include: lack of fluoride exposure, poor socioeconomic status, enamel defects, visible plaque, presence of mutans streptococcus, special health care needs, untreated caries in caregiver or sibling, and frequent/ prolonged exposure to sugary foods/ drinks [38]. Poor nutritional choices and/ or lower SES may very well link obesity and dental caries [38-40]. Despite the potential for an association between obesity and dental caries, our data do not demonstrate either an association between BMI $\mathrm{z}$-score and dental score or a difference in dmft score between normal weight and overweight or obese children. This may be related to the fact that the degree of dental disease was severe in this population studied with a very high mean and median $\mathrm{dmft}$ score. The mean dmft score of 10.0 in this study is in fact higher than those recorded in preschoolers both in the general population and in populations of children with known dental decay [20-25]. The mean dmft score of 5-6 year olds in the 22 wealthiest countries (including the United States) was 1.68 from data collected between 1993 and 2007 [21].

Since data regarding energy and micronutrient intake and their relationship with metabolic profile is limited in this age group as well, we administered the Block Food Frequency questionnaire which is validated for this age group. Our data show that, irrespective of the "weight status", these children's energy intake is inappropriately high, with $51 \%$ of these children consuming over 1400 kilocalories per day, which is the total energy intake recommended by the American Heart Association for 5 year old males, who were the oldest children in the 
population studied. Alarmingly, $29 \%$ of these children consumed more than 1800 kilocalories daily, the energy intake allotted for an adult female. Total daily kilocalories as well as the daily intake of cholesterol, total carbohydrate, fructose, dairy and $100 \%$ juice consumed were positively associated with dmft score. Thus we speculate that high energy diet with poor nutritional content set up a milieu conducive to the development of both "at risk" cardiovascular markers and poor dental health in these young children, many of whom are already overweight. We do have to acknowledge the well-known limitation of all instruments used to assess energy intake and the fact that parental bias may exist [41]. However, it is interesting that most of the time the bias is towards underreporting.

Our data are important as effects of obesity on cardiovascular risk factors have not been previously demonstrated in the under age 5 year group to our knowledge. These data do demonstrate a very high rate of obesity in our population of preschoolers undergoing general anesthesia for full mouth rehabilitation. It also demonstrated an association between increased WCHtR and the inflammatory marker CRP as well as HOMA-IR, a measure of insulin resistance, and significantly increased total and LDLcholesterol in overweight or obese preschool children. Our data also show a significant correlation between WCHtR and BMI z-score, WC, triglycerides, glucose and negative correlation with HDL cholesterol in 2-5 year old children. These are the same metabolic parameters that are altered in the metabolic syndrome, which in adults has been shown to increase risk of type 2 diabetes and increased cardiovascular morbidity and mortality. In children, the criteria for diagnosing metabolic syndrome are greatly debated [32,42]. However, our data suggest that overweight preschoolers are already developing the metabolic syndrome no matter which criteria used [32,42]. These studies need to be replicated in a larger, more diverse population of preschoolers.

\section{Acknowledgements}

KB contributed to the design of the study, collection and analysis of the data. LR contributed to the analysis of the data. PC contributed to the design of the study and collection of the data. PD and HG contributed to the interpretation and performance of laboratory work. AS contributed to the collection and analysis of the data. TQ contributed to the design of the study, collection and analysis of the data. All authors were involved in writing the manuscript and had final approval of the submitted and published versions.

We are very thankful also to the study participants and their parents, to Chris Heard, MD for facilitating the phlebotomy, to Changxing Ma, PhD for statistical help, Sherry Ortiz for help with manuscript preparation and to Robert Borowski, Sara Pannozzo, DDS, Kristin Bender, DDS and Anne Mogavero for assistance in collecting the data. We are grateful for manuscript advice from Teresa Marshall, PhD. This was an investigator initiated project. This study was partially supported by the New York State Department of Health Type 2 Diabetes Center of Excellence grant to T. Quattrin \#C021749.

\section{Declarations}

\section{Ethical approval}

The study was approved by the Children and Youth Institutional Review Board. Written informed consent was obtained from one parent or guardian for all subjects.

\section{References}

1. Flegal KM, Carroll MD, Ogden CL, Curtin LR. Prevalence and Trends in Obesity Among US Adults, 1999-2008. JAMA. 2010; 303(3):235-41. doi: 10.1001/jama.2009.2014.

2. Ogden CL, Carroll MD, Curtin LR, Lamb MM, Flegal KM. Prevalence of high body mass index in US children and adolescents, 2007-2008. JAMA. 2010; 303(3):242-9. doi: 10.1001/jama.2009.2012.

3. Hannon TS, Rao G, Arslanian SA. Childhood obesity and type 2 diabetes mellitus. Pediatrics. 2005; 116(2):473-80.

4. Alberti G, Zimmet P, Shaw J, Bloomgarden Z, Kaufman F, Silink M, et al. Type 2 diabetes in the young: the evolving epidemic: the international diabetes federation consensus workshop. Diabetes Care. 2004; 27(7):1798-811.

5. Franks PW, Hanson RL, Knowler WC, Sievers ML, Bennett PH, Looker HC. Childhood obesity, other cardiovascular risk factors, and premature death. N Engl J Med. 2010; 362(6):485-93. doi: 10.1056/ NEJMoa0904130.

6. Martino F, Puddu PE, Pannarale G, Colantoni C, Martino E, Zanoni C, et al. Hypertension in children and adolescents attending a lipid clinic. Eur J Pediatr. 2013; 172(12):1573-9. doi: 10.1007/s00431-013-20828.

7. The lipid research clinics coronary primary prevention trial results: I. reduction in incidence of coronary heart disease. JAMA. 1984; 251(3):351-64.

8. Stamler J, Wentworth D, Neaton JD. Is relationship between serum cholesterol and risk of premature death from coronary heart disease continuous and graded? Findings in 356,222 primary screenees of the Multiple Risk Factor Intervention Trial (MRFIT). JAMA. 1986; 256(20):2823-8.

9. Berenson GS, Srinivasan SR, Bao W, Newman WP, Tracy RE, Wattigney WA. Association between multiple cardiovascular risk factors and atherosclerosis in children and young adults. The Bogalusa Heart Study. New Engl J Med. 1998; 338(23):1650-6.

10. Jolliffe CJ, Janssen I. Distribution of lipoproteins by age and gender in adolescents. Circulation. 2006; 114(10):1056-62.

11. NCEP Expert Panel on Blood Cholesterol Levels in Children and Adolescents. National Cholesterol Education Program (NCEP): Highlights of the Report of the Expert Panel on Blood Cholesterol Levels in Children and Adolescents. Pediatrics. 1992; 89(3):495-501.

12.Expert Panel on Integrated Guidelines for Cardiovascular Health and Risk Reduction in Children and Adolescents, National Heart, Lung, and Blood Institute. Expert panel on integrated guidelines for cardiovascular health and risk reduction in children and adolescents: summary report. Pediatrics. 2011; 128:S213-56. doi: 10.1542/ peds.2009-2107C.

13.I. Rationale for Attention to Cholesterol Levels in Children and Adolescents. Pediatrics. 1992; 89(3):528-36.

14.O'Neil CE, Keast DR, Nicklas TA, Fulgoni VL. Out-of-hand nut consumption is associated with improved nutrient intake and health 
risk markers in US children and adults: National Health and Nutrition Examination Survey 1999-2004. Nutr Res. 2012; 32(3):185-94. doi: 10.1016/j.nutres.2012.01.005

15. Cullen KW, Watson K, Zakeri I. Relative reliability and validity of the Block Kids Questionnaire among youth aged 10 to 17 years. J Am Diet Assoc. 2008; 108(5):862-6. doi: 10.1016/j.jada.2008.02.015.

16. Smith C, Fila S. Comparison of the Kid's Block Food Frequency Questionnaire to the 24-hour recall in urban Native American youth Am J Hum Biol. 2006; 18(5):706-9.

17. Marshall TA, Eichenberger Gilmore JM, Broffitt B, Stumbo PJ, Levy SM Relative validity of the Iowa Fluoride Study targeted nutrient semiquantitative questionnaire and the block kids' food questionnaire for estimating beverage, calcium, and vitamin D intakes by children. J Am Diet Assoc. 2008; 108(3):465-72. doi: 10.1016/j.jada.2007.12.002.

18. Matthews DR, Hosker JP, Rudenski AS, Naylor BA, Treacher DF, Turner RC. Homeostasis model assessment: insulin resistance and beta-cell function from fasting plasma glucose and insulin concentrations in man. Diabetologia. 1985; 28(7):412-9.

19. Klein H, Palmer CE, Knutson JW. Studies in Dental Caries. Public Health Rep. 1938; 53: 751-65.

20. Phelan C, Byun R, Skinner JC, Blinkhorn AS. Child Dental Health Survey 2007: a snapshot of the oral health status of primary school-aged children in NSW. N S W Public Health Bull. 2009; 20(3-4):40-5. doi: 10.1071/NB08069.

21. Bernabé E, Hobdell MH. Is income inequality related to childhood dental caries in rich countries? J Am Dent Assoc. 2010; 141(2):143-9.

22. Dogar F, Kruger E, Dyson K, Tennant M. Oral health of pre-school children in rural and remote Western Australia. Rural Remote Health. 2011; 11(4):1869.

23. Kruger E, Dyson K, Tennant M. Pre-school child oral health in rural Western Australia. Aust Dent J. 2005; 50(4):258-62.

24. Gizani S, Vinckier F, Declerck D. Caries pattern and oral health habits in 2- to 6-year-old children exhibiting differing levels of caries. Clin Oral Investig. 1999; 3(1):35-40.

25. Dental decay among Australian children. Report no. 53. Australian Institute of Health and Welfare: Canberra, 2011.

26. Barlow SE, Expert Committee. Expert committee recommendations regarding the prevention, assessment, and treatment of child and adolescent overweight and obesity: summary report. Pediatrics. 2007; 120:S164-92.

27. American Academy of Pediatrics. National Cholesterol Education Program: Report of the Expert Panel on Blood Cholesterol Levels in Children and Adolescents. Pediatrics. 1992; 89(3 pt 2):525-84.

28. Ardern CI, Katzmarzyk PT, Janssen I, Ross R. Discrimination of health risk by combined body mass index and waist circumference. Obes Res. 2003; 11(1):135-42.

29. Katzmarzyk PT, Janssen I, Ross R, Church TS, Blair SN. The importance of waist circumference in the definition of metabolic syndrome: prospective analyses of mortality in men. Diabetes Care. 2006 29(2):404-9.
30. Freedman DS, Dietz WH, Srinivasan SR, Berenson GS. Risk factors and adult body mass index among overweight children: the Bogalusa Heart Study. Pediatrics. 2009; 123(3):750-7.

31. Maffeis C, Banzato C, Talamini G. Waist-to-Height Ratio, a Useful Index to Identify High Metabolic Risk in Overweight Children. J Pediatr. 2008; 152(2): 207-13. doi: 10.1016/j.jpeds.2007.09.021.

32. Martino F, Puddu PE, Pannarale G, Colantoni C, Zanoni C, Martino E, et al. Metabolic syndrome among children and adolescents from Southern Italy: Contribution from the Calabrian Sierras Community Study (CSCS). Int J Cardiol. 2014; 177(2):455-60. doi: 10.1016/j. ijcard.2014.09.020.

33. Skinner AC, Steiner MJ, Henderson FW, Perrin EM. Multiple markers of inflammation and weight status: cross-sectional analyses throughout childhood. Pediatrics. 2010; 125(4):e801-9. doi: 10.1542/peds.20092182.

34. Ford ES, Giles WH, Myers GL, Rifai N, Ridker PM, Mannino DM. C-reactive protein concentration distribution among US children and young adults: findings from the National Health and Nutrition Examination Survey, 1999-2000. Clin Chem. 2003; 49(8):1353-7.

35. Ford AL, Hunt LP, Cooper A, Shield JP. What reduction in BMI SDS is required in obese adolescents to improve body composition and cardiometabolic health? Arch Dis Child. 2010; 95(4):256-61. doi: 10.1136/adc.2009.165340.

36. Singh GK, Siahpush M, Kogan MD. Rising social inequalities in US childhood obesity, 2003-2007. Ann Epidemiol. 2010; 20(1):40-52. doi: 10.1016/j.annepidem.2009.09.008.

37. Singh GK, Kogan MD, Van Dyck PC, Siahpush M. Racial/Ethnic, Socioeconomic, and Behavioral Determinants of Childhood and Adolescent Obesity in the United States: Analyzing Independent and Joint Associations. Ann Epidemiol. 2008; 18(9):682-95. doi: 10.1016/j.annepidem.2008.05.001

38. Tinanoff N, Reisine S. Update on Early Childhood Caries Since the Surgeon General's Report. Acad Pediatr. 2009; 9(6):396-403. doi: 10.1016/j.acap.2009.08.006

39. Vargas CM, Crall JJ, Schneider DA. Sociodemographic Distribution of Pediatric Dental Caries: NHANES III, 1988-1994. J Am Dent Assoc. 1998; 129(9):1229-38.

40. Marshall TA, Eichenberger-Gilmore JM, Broffitt BA, Warren JJ, Levy SM. Dental caries and childhood obesity: roles of diet and socioeconomic status. Community Dent Oral Epidemiol. 2007;35(6):449-58.

41. Livingstone MB, Robson PJ, Wallace JM. Issues in dietary intake assessment of children and adolescents. Br J Nutr. 2004;92:S213-22.

42. Cook S, Auinger P, Li C, Ford ES. Metabolic Syndrome Rates in United States Adolescents, from the National Health and Nutrition Examination Survey, 1999-2002. J Pediatr. 2008; 152(2):165-70. doi: 10.1016/j.jpeds.2007.06.004 